\title{
Páncreas ectópico en antro gástrico
}

\author{
José Fernando Hernández Amador, ${ }^{1}$ Mario Alberto Arredondo Ortiz, ${ }^{1}$ Eduardo Villanueva Sáenz, ${ }^{2}$ \\ Catalina Romo Aguirre, ${ }^{3}$ Carlos Benjamín González Sánchez ${ }^{4}$
}

\section{Resumen}

El páncreas ectópico presenta una incidencia baja. Predomina en el sexo masculino entre la quinta y sexta década de vida y se localiza habitualmente en el estómago, por lo regular cursa asintomático dependiendo de su tamaño. Es posible que se manifieste mediante epigastralgia u obstrucción gástrica. El diagnóstico de certeza es difícil; la endoscopia con biopsia, tomografía y ultrasonido endoscópico con biopsia con aguja fina pueden ayudar a llegar a éste. Actualmente no se cuenta con un tratamiento consensado y puede realizarse desde una resección endoscópica hasta diversos tipos de gastrectomías.

Palabras clave: Tumor gástrico, páncreas ectópico, Y de Roux, antrectomía.

\section{INTRODUCCIÓN}

El páncreas ectópico es la segunda anomalía congénita pancreática más frecuente después del pancreas divisum. Descrito por primera vez por Jean Schultz en 1729, se define como la presencia de tejido pancreático que carece de comunicación anatómica o vascular con el cuerpo principal del páncreas. ${ }^{1}$ Se manifiesta con más frecuencia hacia la quinta o sexta década de la vida y tiene un pre-

\footnotetext{
${ }^{1}$ Residente de cirugía general, Facultad Mexicana de Medicina, Universidad La Salle.

${ }^{2}$ Cirugía gastrointestinal y colorrectal.

3 Anatomía patológica.

${ }^{4}$ Gastroenterología y endoscopia.
}

Hospital Ángeles Pedregal.

Correspondencia:

Dr. E Villanueva-Sáenz

Correo electrónico: dredevilla@me.com

Aceptado: 09-06-2016.

Este artículo puede ser consultado en versión completa en http:// www.medigraphic.com/actamedica

\section{Summary}

Ectopic pancreas is a rare entity. Usually found in $50-60$ year old males, its most frequent location is the stomach; frequently asymptomatic, depending on the size of the tumor it could manifest with epigastric pain or occlusion. Obtaining a definitive diagnosis is difficult preoperatively. Diagnostic methods include endoscopic biopsy, tomography and endoscopic ultrasound with fine-needle biopsy, although their role is limited. So far there is no consensus for the treatment of choice in ectopic pancreas, which ranges from endoscopic resection to diverse gastrectomy techniques.

Key words: Gastric tumor, ectopic pancreas, Roux-en-Y, antrectomy.

dominio de incidencia en hombres. ${ }^{1-3}$ La localización más frecuente es en el estómago (25-38\% de los casos), seguido de duodeno, yeyuno e íleon, es probable que se presente en otros órganos abdominales como colon, vesícula biliar, hígado, bazo, epiplón, en un divertículo de Meckel, entre otros. ${ }^{4-6}$ Se producen síntomas en $40 \%$ de los casos, el resto se diagnostican incidentalmente.

\section{PRESENTACIÓN DEL CASO}

Paciente femenino de 55 años de edad, sin antecedentes médico-quirúrgicos relevantes que acudió por cuadro de epigastralgia ardorosa intermitente, plenitud, pirosis y regurgitación de tres años de evolución. Se realizó protocolo de estudio para H. pylori, resultando positivo. Se solicitó panendoscopia en la que se detectó una tumoración submucosa prepilórica de $2.5 \mathrm{~cm}$ de diámetro con bordes regulares y bien definidos (Figura 1). La biopsia de la lesión reportó gastritis folicular asociada a H. pylori, sin otros datos concluyentes. La tomografía simple de abdomen mostró una lesión gástrica submucosa antral con hipodensidad central, sin adenopatías locorregionales (Figura 2). Se indicó esquema erradicador de $H$. pylori y a petición de la paciente se decidió manejo expectante con control edoscópico anual, sin reportarse cambios morfológicos 

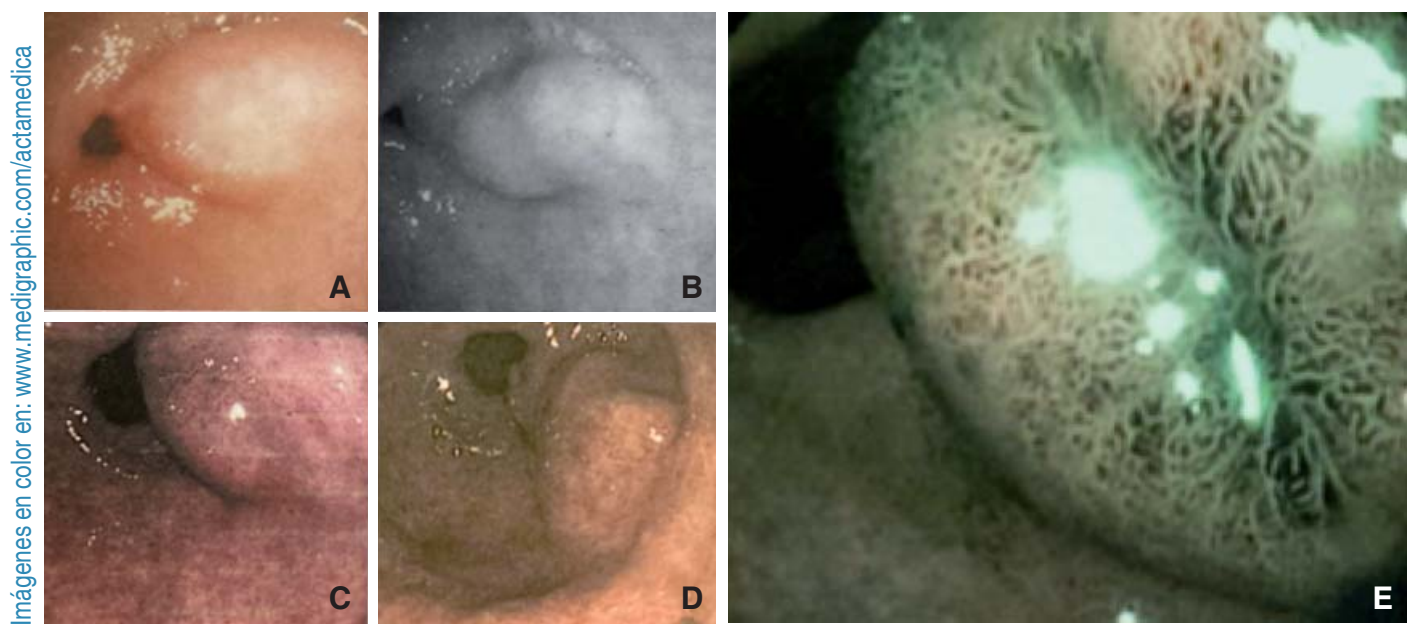

Figura 1.

Control endoscópico: A. Abril 2007, B. Diciembre 2008, C. Marzo 2011, D. Mayo 2013, E. Marzo 2015.
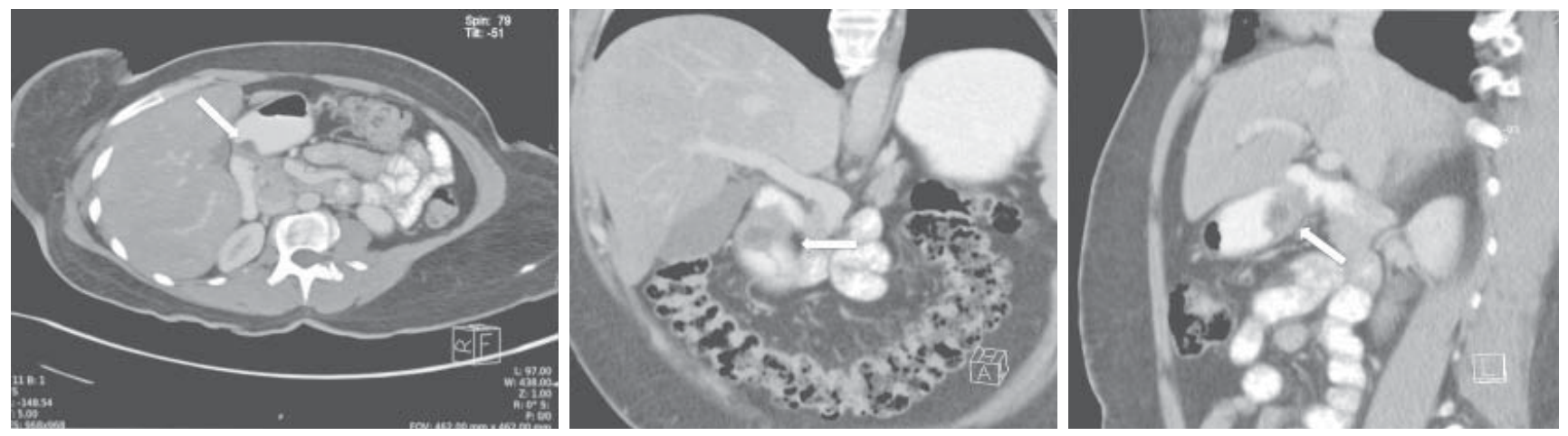

Figura 2. Tomografía axial computarizada de abdomen con contraste oral en la que se observa lesión prepilórica submucosa de $2.5 \mathrm{~cm}$ con hipodensidad central (flechas).

de la lesión en los estudios subsecuentes. La paciente continuó con sintomatología a pesar de manejo adecuado con medidas higiénico-dietéticas, inhibidor de bomba de protones y procinéticos. Al sexto año de vigilancia presentó exacerbación de los síntomas, así como crecimiento de la lesión en endoscopia de control (Figura 1). Se decidió realizar antrectomía más reconstrucción con gastro-yeyuno anastomosis en Y-de-Roux y vagotomía troncular mediante abordaje laparoscópico (Figura 3). El estudio histopatológico de la lesión reportó la presencia de un páncreas heterotópico submucoso antral, de $3.5 \mathrm{~cm}$ de diámetro, con componente quístico, con zonas de inflamación aguda severa y crónica (Figura 3). La paciente tuvo una evolución postoperatoria favorable y fue egresada al quinto día de hospitalización, con remisión completa de los síntomas.

\section{DISCUSIÓN}

El páncreas ectópico es una entidad poco frecuente. Su incidencia reportada en series de autopsias varía entre 0.5 y $13.7 \%$. Su localización más habitual es el estómago, hasta $95 \%$ se encuentra a nivel de antro y hasta $73 \%$ es submucoso. ${ }^{1,7}$ Los diagnósticos diferenciales son: linfoma, tumor de estroma gastrointestinal (GIST), carcinoide o adenocarcinoma gástrico. La endoscopia suele mostrar una lesión de base ancha y bordes bien definidos que se encuentra umbilicada en alrededor de $50 \%$ de los casos. Su situación submucosa ocasiona dificultad para obtener una biopsia adecuada por vía endoscópica, resultando en diagnósticos no concluyentes. Otros recursos diagnósticos son la tomografía axial computarizada con contraste en la que puede observarse un refuerzo de la lesión similar a la del tejido pancreático normal o el ultrasonido endoscópico que permite la realización de biopsia por aspiración con aguja fina. ${ }^{1,2}$

Existen dos hipótesis respecto a su etiología: 1) La implantación de tejido pancreático embriónico al momento de la rotación intestinal en la sexta semana de gestación y 2) la metaplasia pancreática inapropiada de tejido endodérmico durante la embriogénesis. ${ }^{1}$ La clasificación de Heinrich, modificada por Gaspar y Fuentes, distingue 

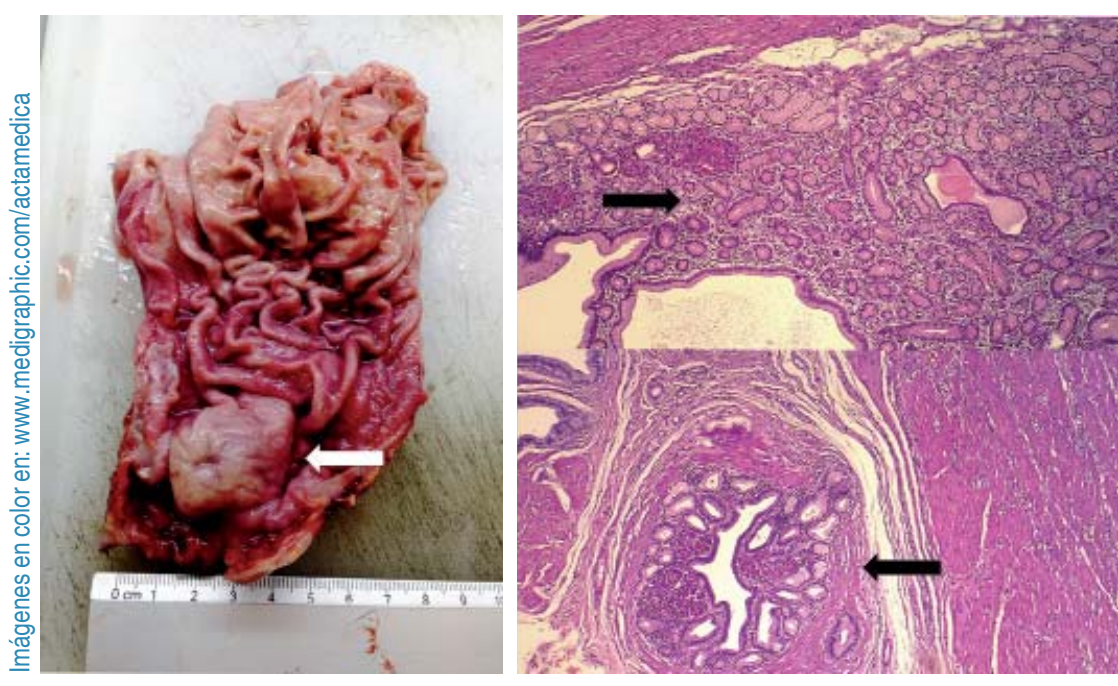

Figura 3.

Patología. Pieza quirúrgica producto de antrectomía con tumoración prepilórica umbilicada (páncreas ectópico) y cortes histológicos con evidencia de ductos, acinos e islotes pancreáticos (tejido pancreático típico, Heinrich tipo I). cuatro tipos de ectopia pancreática de acuerdo con su composición histológica: tipo 1: tejido pancreático típico (heterotópico) con acinos, ductos e islotes (heterotópico); tipo 2: sólo ductos pancreáticos; tipo 3: sólo tejido acinar y tipo 4: sólo islotes (páncreas endocrino). ${ }^{8}$

Los síntomas más frecuentes son epigastralgia, dispepsia, plenitud y pirosis. La hemorragia, la inflamación aguda o crónica y la formación de pseudoquistes son poco frecuentes. El índice de malignización de estas lesiones es bajo (1.8\%). ${ }^{1-5,8}$

No existe consenso con respecto a las indicaciones del manejo quirúrgico en lesiones pequeñas y asintomáticas. Se ha reportado la disección submucosa por vía endoscópica de este tipo de tumores. Se recomienda este abordaje en lesiones pequeñas (menores de $2 \mathrm{~cm}$ ) por ser menos invasivo, porque no deforma la anatomía y porque permite un diagnóstico histopatológico preciso. Los riesgos asociados a este procedimiento son sangrado, perforación e infección intraabdominal. No debe realizarse en lesiones adheridas a la capa muscular. ${ }^{8}$ La cirugía se indica en pacientes sintomáticos, en lesiones mayores de $3 \mathrm{~cm}$ ( $>1.5 \mathrm{~cm}$ en tumores prepilóricos por el riesgo de obstrucción del tracto de salida gástrico), para confirmación diagnóstica en caso de biopsias endoscópicas inadecuadas o ante la sospecha de malignidad. ${ }^{2,9}$

\section{REFERENCIAS}

1. Lai EC, Tompkins RK. Heterotopic pancreas. Review of a 26-year experience. Am J Surg. 1986; 151 (6): 697-700.

2. Ormarsson OT, Gudmundsdottir I, Mårvik R. Diagnosis and treatment of gastric heterotopic pancreas. World J Surg. 2006; 30 (9): 16821689.

3. Tanaka K, Tsunoda T, Eto T, Yamada M, Tajima Y, Shimogama $\mathrm{H}$ et al. Diagnosis and management of heterotopic pancreas. Int J Surg. 1993; 78 (1): 32-35.

4. Baisakh MR, Nachiketa M, Baisakh P, Sahoo S, Kar M. Heterotopic pancreas in the stomach masquerading neoplasm. J Clin Diagn Res. 2014; 9 (1): FL01-2.

5. Rodríguez Aa, Berquist W, Bingham D. Gastric outlet obstruction caused by heterotopic pancreas in an adolescent. Dig Dis Sci. 2015; 60 (2): 835-837.

6. Inoue Y, Hayashi M, Arisaka Y, Higuchi K, Egashira Y, Tanigawa N. Adenocarcinoma arising in a heterotopic pancreas (Heinrich type III): a case report. J Med Case Rep. 2010; 4 (1): 39.

7. Hernández M, Rico A, Qazi O. Gastric heterotopic pancreas presenting as a submucosal tumor in a 27 -year-old male. ACG Case Rep J. 2014; 2 (1): 8-9.

8. Zhang y, Huang Q, Zhu LH, Zhou LP, Mao XL. Endoscopic excavation for gastric heterotopic pancreas: an analysis of 42 cases from a tertiary center. Wien Klin Wochenschr. 2014; 126 (3): 509-514.

9. Ayantunde AA, Pinder E, Heath DI. Symptomatic pyloric pancreatic heterotopia: Report of three cases and review of the literature. Med Sci Monit. 2006; 12 (6): CS49-52. 